\title{
Parametric Dependence of Coordination Number Obtained Using Derjaguin, Landau, Verwey and Overbeek DLVO Pair Interaction Potential for Ordered Macroions Suspended into Water
}

\author{
Rakesh Kumar Pandey \\ Department of Physics, Synod Higher Secondary School, Mission Vengthlang, Aizawl, India \\ Email: drrakesh0107@yahoo.co.in
}

Received 2 June 2014; revised 12 July 2014; accepted 19 August 2014

Copyright (C) 2014 by author and OALib.

This work is licensed under the Creative Commons Attribution International License (CC BY). http://creativecommons.org/licenses/by/4.0/

(c) (i) Open Access

\begin{abstract}
Coordination numbers have been calculated for the ordered structures prevailing in aqueous suspension of macroions for its different governing parameters viz. sizes, charges, impurity ion concentrations and temperatures by integration of region $r^{2} g(r)$ in the limit $0-r_{\min }$ around the main peak in $g(r)-r$ curve in order to examine the effects of controlling parameters on the coordination number. The failure of $R M S A$ method has been reported in case of variation of temperature and effective charge over macroions in terms of volume fraction. Orderings of $F C C / H C P$ type with coordination number nearly 12 amongst the macroions suspended into water have been reported to exist.
\end{abstract}

\section{Keywords}

DLVO Potential, Coordination Number and Ordered Structures

Subject Areas: Applied Physics, Condensed State Physics

\section{Introduction}

Macroionic suspension contains particles sizes [1]-[3] ranging $50 \AA$ - 10,000 $\AA$ with about ionizable surface charges $10^{2}-10^{5}$ per particle [4] in electronic unit. These particles dissociate into a suitable solvent like water and provide large electrostatic negative charges per particle. These negatively charged particles, called ma- 
croions [2] [5], arrange themselves in the suspension due to their electrostatic interactions. Mainly the repulsive coulomb screened pair potential i.e., Derjaguin, Landau, Verwey and Overbeek DLVO pair potential [6] developed between suspended macroions due to their surface charges monitors the arrangement of macroions in the suspension. Depending on strength of $D L V O$ pair interaction potential, ordering i.e., gas-, liquid- and solid-like structures prevail in the macroionic suspension [7]-[24]. As ordering amongst the suspended macroions develops, pair correlation function $g(r)$ versus distance $r$ curve bestows peaks. Integration of region $r^{2} g(r)$ in the limit 0 $r_{\min }$ around the first main peak in $g(r)-r$ curve approximates the coordination number $N$ i.e. total number of the nearest macroions surrounding an individual macroions [7] [8].

Concentrations of macroions $\rho$ and impurity ions $\rho_{i}$, charges over the macroions $z$ and impurity ions $z_{i}$, dielectric constant $\varepsilon$, sizes $\sigma$ and absolute temperature $T$ of solvent are the governing parameters of the pair correlation function through the pair interaction potential and hence these parameters control the coordination number [11] [12]. Tata et al. [7] have calculated the coordination number for different concentrations of impurity ions to study the effect of impurity ions on ordered structures prevailing in aqueous macroionic suspension using the method of integration of region $r^{2} g(r)$. Pandey and Tripathi [8] have calculated the coordination numbers using this method to study the ordered behavior of binary suspension of macroions by changing controlling parameters of the suspension. Recently, Pandey has used the integration method of region $r^{2} g(r)$ in the limit $0-r_{\min }$ to calculate the coordination number in order to ascertain the ordering nature in the plasmas [9] and biomacroions [10]. The technique of integration of region $r^{2} g(r)$ in the limit $0-r_{\min }$ however, estimates somewhat higher numerical values of coordination number in case of liquid like ordering than solid structures, and endows an idea regarding the arrangement of macroions in the suspension [7]-[12]. It is an easy approach to estimate the coordination number under the theoretical considerations in order to predict the type of ordering prevailing in aqueous macroionic suspension if measurements of lattice parameters are not available or possible.

From the literatures survey, it is found that the structural behavior in aqueous suspension of monomacroions has not been studied properly in the light of coordination number for its different controlling parameters viz. sizes, charges, impurity ion concentrations and temperatures [1]-[32]. Therefore, it becomes quite imperative to calculate coordination number for various governing parameters to ascertain the ordering nature of macroionic suspensions. In the present theoretical work, coordination number has been calculated by integrating the region $r^{2} g(r)$ in the limit $0-r_{\min }$ around the first main peak obtained in $g(r)-r$ curve to study the effect of above controlling parameters on it as well as to ascertain the nature of orderings prevailing amongst the suspended macroions into water. The pair correlation function $g(r)$ data have been obtained from the Fourier transform of structure factor $S(Q)$ data computed with rescaled mean spherical approximation RMSA method employing DLVO pair interaction potential between suspended macroions [13] [14]. Close packed ordered structures amongst the macroions suspended into water with coordination number nearly 12, indicating FCC/HCP type of structure has been found and reported.

\section{Theory}

The DLVO pairing interaction potential between macroions [6] in its dimensionless form is

$$
\beta U(x)=\gamma \exp (-k x) / x, x \geq 1 ;
$$

where $x=r / \sigma, k=\kappa \sigma, \beta=1 / k_{B} T$ and $\kappa$ is the Debye-Hückel inverse screening length given by

$$
\begin{gathered}
\kappa^{2}=\left(\rho z+\sum \rho_{i} z_{i}\right) e^{2} / k_{B} T \varepsilon, \\
\text { and } \gamma=\beta \pi \varepsilon \sigma \psi_{o}^{2} \exp (k)
\end{gathered}
$$

is dimensionless coupling constant and its value is $\gamma \gg 1$ in macroionic suspension [2] and the expression of surface potential $\psi_{o}$ of each macroion [13] [14] is

$$
\psi_{o}=z e /(\pi \varepsilon \sigma(2+k)) \text {. }
$$

The contact potential between pairs of macroions in unit of $k_{B} T$ is

$$
\gamma \exp (-k)=\beta \pi \varepsilon \sigma \psi_{o}^{2}
$$


The Ornstein-Zernike OZ equation [25] given by

$$
h(x)=c(x)+\rho \sigma^{3} \int_{0}^{\infty} h(x-y) c(x) d x
$$

relates total correlation function $h(x)=g(x)-1$ and direct correlation function $c(x)$ together with mean spherical approximation

$$
\begin{gathered}
h(x)=-1, x<1 ; \\
c(x)=-\beta U(x), x>1 ;
\end{gathered}
$$

help defining the structure factor

$$
S(Q)=1+12 \eta \int_{0}^{\infty} h(x) \exp (-i k x) d x
$$

where $\eta=\pi \rho \sigma^{3} / 6$ is volume fraction of macroions and $Q$ is dimensionless momentum transfer.

Fourier Transform of $S(Q)$ gives pair correlation function $g(x)$ [27]

$$
g(x)=1+\frac{1}{12 \eta \pi x} \int_{o}^{\infty}[S(Q)-1] Q \sin (Q x) d Q
$$

Coordination number can be obtained using relation [7]-[12]

$$
N=24 \eta \int_{0}^{x_{\min }} x^{2} g(x) d x
$$

where $x_{\min }$ is the first minimum in $g(x)-x$ curve.

Structure factors have been calculated using program developed by Hayter and Hansen [26] for the input of pair correlation function calculated using a program developed by Hayter [27] to get the input data for the coordination number. Coordination numbers have been calculated by self-developed computer program in FORTRAN-77 using Equation (11), which has already been tested for its accuracy elsewhere [8]-[12].

\section{Results and Discussion}

Figure 1 shows the variation of pair correlation function with dimensionless distance for the system $\rho=4.0 \times$ $10^{18} \mathrm{~m}^{-3}, \sigma=800 \AA, T=294 \mathrm{~K}, \varepsilon=78.5$ for charges $\mathrm{z}=600 \mathrm{e}$. The Coordination number has been calculated by integrating the region $x^{2} g(x)$ in the limit $0-x_{\min }$ around the first main peak obtained in $g(x)$ - $x$ curve as shown in Figure 1.

In order to examine the effect of charge over macroions suspended into water on coordination number $N$, the coordination numbers have been calculated for the macroionic suspension of parameters $\rho=4.0 \times 10^{18} \mathrm{~m}^{-3}, \sigma=$ $800 \AA, T=294 \mathrm{~K}, \varepsilon=78.5$ for charges $\mathrm{z}=600 \mathrm{e}, 800 \mathrm{e}, 1000 \mathrm{e}$, which are given in Table 1 . The value of $N$ increases with charge increase over macroions. It is because, increase in the charge over macroions increases the intermacroionic pairing interaction potential that results an increase in the bond strength between ordered macroions. Thus, the bond lengths between ordered macroions contract, which cause more macroions to come close to take part in the ordering and thereby increase the value of $N$ with increase in charge over macroions [8]-[12].

Table 2 contains the coordination numbers for the aqueous suspension of macroions of parameters $\rho=4.0 \times$ $10^{18} \mathrm{~m}^{-3}, \sigma=872 \AA, z=440 \mathrm{e}, \varepsilon=78.5$ for temperatures $T=294 \mathrm{~K}, 313 \mathrm{~K}, 323 \mathrm{~K}, 333 \mathrm{~K}$ and $343 \mathrm{~K}$. It is clear that $N$ remains nearly same with increase in temperature of suspension. This indicates that temperature does not affect appreciably the nature of pair correlation function via pair interaction potential and thus, $N$ remains almost unaltered with increase in the temperature in RMSA calculation.

The results of present investigations, not expected, are in disagreement with the experimental results reported by Schaefer [28] wherein temperature has been found to play naturally a significant role in the ordering and disordering phenomenon occurring in macroionic suspension, which leads to a complete disappearance of peaks in $g(x)-x$ curve and thence lessen the coordination number with increase in temperature. Dielectric constant of water, as it is a polar solvent, decreases with increase in temperature. This causes to decrease in the degree of dis- 


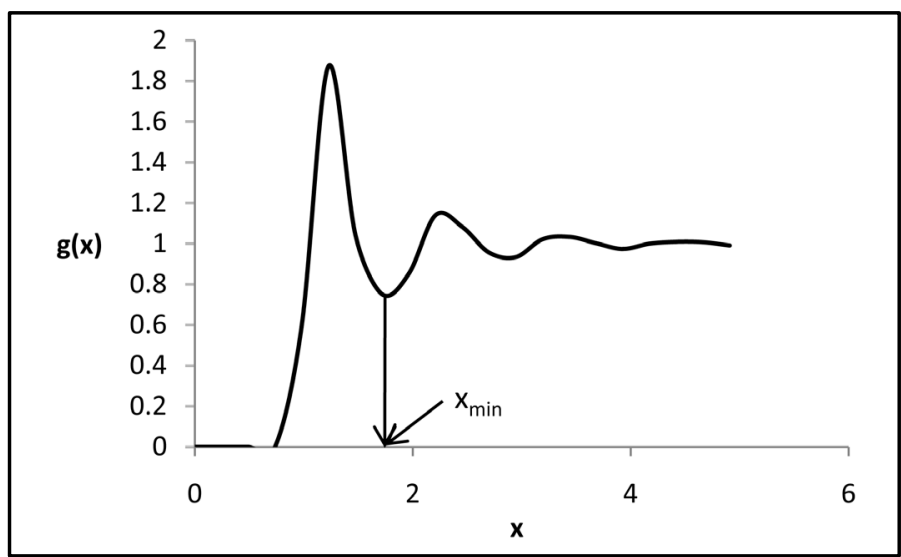

Figure 1. Variation of pair correlation function with dimensionless distance for the system of $\rho=4.0 \times 10^{18} \mathrm{~m}^{-3}, \sigma=800 \AA, T=294 \mathrm{~K}, \varepsilon$ $=78.5$ for charges $z=600$ e.

Table 1. Variation of coordination number with charge for the system of $\rho=4.0 \times 10^{18} \mathrm{~m}^{-3}, \sigma=800 \AA, \varepsilon=78.5$ and $T=294 \mathrm{~K}$.

\begin{tabular}{cc}
\hline No. of charges $z$ & Coordination number $N$ \\
\hline 600 & 13.1 \\
800 & 13.5 \\
1000 & 14.0 \\
\hline
\end{tabular}

Table 2. Variation of coordination number with temperature for the system of $\rho=4.0 \times 10^{18} \mathrm{~m}^{-3}, \sigma=872 \AA, z=440$ e and $\varepsilon=78.5$.

\begin{tabular}{cc}
\hline Temperature $T(\mathrm{~K})$ & Coordination number $N$ \\
\hline 294 & 17.2 \\
313 & 17.2 \\
323 & 17.2 \\
333 & 17.2 \\
343 & 17.2 \\
\hline
\end{tabular}

sociation of particles into solvent and produces less value of charge per macroion. Thermal energy per macroion also increases with increase in temperature of solution. The decrease in number of charge over each macroion and increase in its thermal energy with increasing temperature results decrease in the pairing interaction potential between them and hence weakening the bond strength between them. Under these circumstances, each macroion therefore, tries to follow Brownian movement due to melting of crystalline order of suspended macroions on increasing the temperature and hence shows poor ordering in the Schaefer's experiment on ordering of macroionic suspension. This behavior is not found in the present calculation using RMSA method for the same parameters. As the RMSA method does not include temperature dependence of dielectric constant, so it is of no use to present the discussion about the results in Table 2 . However, present study suggests some modifications in the RMSA method to replicate results of Schaefer's experiments of temperature dependence of ordering in the suspended macroions [8]-[12].

The effect of concentrations of impurity ions on the coordination number has been studied for the aqueous system of macroions of $\rho=4.0 \times 10^{18} \mathrm{~m}^{-3}, \sigma=1090 \AA, z=600 \mathrm{e}, \varepsilon=78.5, T=294 \mathrm{~K}$ for different impurity ions concentrations $\rho_{i}=0.0,0.001 z \rho, 0.01 z \rho, 0.5 z \rho, z \rho, 1.5 z \rho, 2 z \rho$ and $3 z \rho$. The coordination numbers obtained for these parameters are in Table 3 . The coordination number decreases gradually with increase in impurity ion 
concentration. Tata et al. [7] have also reported the same behavior with increase in concentrations of impurity ions. The increase in impurity ion concentration reduces interaction potential and screening length between macroions, which leads melting of the crystalline order. Addition of ionic impurity ions concentration reduces coulomb screening length and thus $g(x)$ smears out with shorten of the peaks in $g(x)$-x curve that gives scanty value of coordination number.

Coordination number for the suspended macroions of $\rho=4.0 \times 10^{18} \mathrm{~m}^{-3}, \varepsilon=78.5, \sigma=800 \AA, 1090 \AA, 1200$ $\AA$ into water at temperature $T=294 \mathrm{~K}$ are given in the Table 4 for $z=500$ e and for $z=800$ e in the Table 5 . The coordination number decreases with increase in the size of the macroions. This behavior may be explained in terms of effective volume fraction [29] $\eta=\eta^{\text {eff }}(1+2 / k)^{3}$. Intermacroionic distance remains unaltered at fixed concentration of macroions. However, surface-to-surface distance between macroions decreases with increase in their sizes and therefore, causes to increase in effective volume fraction that results decrease in pair interaction potential due to decrease in surface potential [15] [16]. In turn, peaks in $g(x)-x$ curve shrink in their width and enhance in their heights that indicate the weakening of binding strength between intermacroionic layers with increase in interlayer distance and thereby $N$ decreases due to decrease in correlation length between macroions. In another approach as pointed out by Wagner et al. [30] the effective charges $z^{\text {eff }}=z /(1-\eta)$, increases with increase in size of macroions which gives rise to increase in intermacroionic pairing interaction potential and then to correlation length. Because of this, more macroions would come close to take part in ordering and therefore, $N$ should increase with size increase of macroions. Instead, it decreases in the present calculation. Thus, present result is again in contrary if explained in terms of effective charge.

\begin{tabular}{|c|c|}
\hline Impurity ions $\rho_{i}(\times z \rho)$ & Coordination number $N$ \\
\hline 0.000 & 15.5 \\
\hline 0.001 & 15.3 \\
\hline 0.010 & 15.2 \\
\hline 0.500 & 14.6 \\
\hline 1.000 & 14.0 \\
\hline 1.500 & 13.7 \\
\hline 2.000 & 13.4 \\
\hline 3.000 & 12.3 \\
\hline
\end{tabular}

Table 4. Variation of coordination number with size for the system of $\rho$ $=4.0 \times 10^{18} \mathrm{~m}^{-3}, \varepsilon=78.5, T=294 \mathrm{~K}$ and $z=500 \mathrm{e}$.

\begin{tabular}{cc}
\hline Sizes $(\AA)$ & Coordination number $N$ \\
\hline 800 & 12.9 \\
1090 & 12.6 \\
1200 & 12.4 \\
\hline
\end{tabular}

\begin{tabular}{|c|c|}
\hline Sizes $(\AA)$ & Coordination number $N$ \\
\hline 800 & 13.5 \\
\hline 1090 & 13.2 \\
\hline 1200 & 12.7 \\
\hline
\end{tabular}




\section{Conclusion}

Coordination number depends linearly on the charge in inverse with impurity ions concentration and size of macroions. The results of the present calculation disclose the failure of RMSA in the case of temperature variation as well in terms of effective charge over macroions and thus, RMSA requires some modifications to make it enable to reproduce the experimental results with variation of temperature and effective charge. Ordered structures of coordination number nearly 12 indicating FCC/HCP types of ordering prevail in the aqueous macroionic suspension. In order to ascertain either FCC or HCP types of structures, an experiment needs to be performed to measure the intermacroionic layer distances i.e., the lattice constants.

\section{Acknowledgements}

Author is grateful to Profs. D. N. Tripathi, S. N. Thakur and K. N. Upadhyay for their valuable supports and encouragements.

\section{References}

[1] Brown, J.C., Pusey, P.N., Goodwin, J.W. and Ottewill, R.H. (1975) Light Scattering Study of Dynamic and Time Averaged Correlations in Dispersions of Charged Particles. Journal of Physics A: Mathematical and General, 8, 664-682.

[2] Hayter, J.B. (1985) Physics of Amphiphiles: Micelles, Vesicles and Microemulsions. In: Degiorgio, V. and Corti, M., Eds., Proceeding of the International School of Physics, North Holland, Amsterdam, 59.

[3] Pandey, R.K. (2014) Effect of Solvents on the Degree of Ionization of Polystyrene Balls and Their Liquid Crystallization in the Suspension Using Static Light Scattering Method. SOP Transactions on Physical Chemistry, 1, 29-40.

[4] Alexander, S., Chaikin, P., Grant, P., Morales, G., Pincus, P. and Hone, D. (1984) Charge Renormalization, Osmotic Pressure and Bulk Modulus of Colloidal Crystals: Theory. The Journal of Chemical Physics, 80, 5776-5781. http://dx.doi.org/10.1063/1.446600

[5] Pandey, R.K. and Tripathi, D.N. (2010) Comparison of Structure Factors and Pair Correlation Functions Obtained Using Coulomb Attractive Potential and Screened Coulomb Potential for the Suspended Macroions. Indian Journal of Physics B, 84, 397-404. http://dx.doi.org/10.1007/s12648-010-0024-0

[6] Verwey, E.J.W. and Overbeek, J.Th.G. (1948) Theory of the Stability of Lyophobic Colloids: The Interaction of Sol Particles Having an Electric Double Layer. Elsevier, Amsterdam.

[7] Tata, B.V.R., Kesavamoorthy, R. and Arora, A.K. (1986) Onrmsa Description of Liquid Order in Dilute Charged Colloidal Suspensions. Molecular Physics, 57, 369-378. http://dx.doi.org/10.1080/00268978600100291

[8] Pandey, R.K. and Tripathi, D.N. (2001) Schulz Distribution Function and the Polydispersity of the Binary Suspension of Charged Macroions. Colloids and Surfaces A: Physicochemical and Engineering Aspects, 190, 217-227. http://dx.doi.org/10.1016/S0927-7757(01)00707-5

[9] Pandey, R.K. (2014) Comparison of Pair Correlation Functions Obtained Using SI and DLVO Potentials for the Ordered Plasmas. SOP Transactions on Theoretical Physics, 1, 101-109.

[10] Pandey, R.K. (2014) Liquid Crystals in Spheroid Macroions and Spherocylindrical Biomacroions Suspended into Water Using Sogami-Ise Potential. SOP Transactions on Applied Physics, 1, $27-37$.

[11] Pandey, R.K. (1998) A Study of Ordering in Macroionic Suspension and Related Problems. Ph.D. Thesis, Banaras Hindu University, Varanasi.

[12] Pandey, R.K. (2013) Ordering in Suspended Charged Particles. Lambert Academic Publishing, Saarbrücken.

[13] Hayter, J.B. and Penfold, J. (1981) An Analytic Structure Factor for Macroion Solutions. Molecular Physics, 42, 109118. http://dx.doi.org/10.1080/00268978100100091

[14] Hayter, J.B. and Hansen, J.P. (1982) A Rescaled Mean Spherical Approximation Structure Factor for Dilute Charged Colloidal Dispersion. Molecular Physics, 46, 651-656. http://dx.doi.org/10.1080/00268978200101471

[15] Pandey, R.K. and Tripathi, D.N. (1992) Rescaled Mean Spherical Approximation Structure Factor for an Aqueous Suspension of Polystyrene Spheres. Pramana Journal of Physics, 39, 589-595. http://dx.doi.org/10.1007/BF02847278

[16] Pandey, R.K. and Tripathi, D.N. (1995) Effect of Charge and Size on Ordering of Highly Charged Concentrated Macroions in Suspension. Pramana Journal of Physics, 44, 1-7. http://dx.doi.org/10.1007/BF02898207

[17] Härtl, W. and Versmold, H. (1984) Temperature Dependence of the Structure Factor S(Q) of Liquid-Like Ordered Colloidal Dispersions. The Journal of Chemical Physics, 81, 2507-2510. http://dx.doi.org/10.1063/1.447911

[18] Nagele, G., Medina-Noyola, M., Klein, R. and Arauz-Lara, J. (1988) Time-Dependent Self-Diffusion in Model Sus- 
pensions of Highly Charged Brownian Particles. Physica A: Statistical Mechanics and Its Applications, 149, 123-163.

[19] Aguanno, B.D. and Klein, R. (1991) Structural Effects of Polydispersity in Charged Colloidal Dispersions. Journal of Chemical Society Faraday Transactions, 87, 379-390. http://dx.doi.org/10.1039/ft9918700379

[20] Ruiz-Estrada, H., Medina-Noyola, M. and Nagele, G. (1990) Rescaled Mean Spherical Approximation for Colloidal Mixtures. Physica A: Statistical Mechanics and Its Applications, 168, 919-941.

[21] Krause, R., Nagele, G., Karrer, D., Schneider, J., Klein, R. and Weber, R. (1988) Structure and Self-Diffusion in Dilute Suspensions of Polystyrene Spheres: Experiment vs. Computer Simulation and Theory. Physica A: Statistical Mechanics and Its Applications, 153, 400-419.

[22] Kesavamoorthy, R. and Arora, A.K. (1986) Elastic Constants of Colloidal Glass. Journal of Physics C: Solid State Physics, 19, 2833-2846.

[23] Tata, B.V.R., Kesavamoorthy, R. and Sood, A.K. (1987) Structure Factor for a Two Component Mixture of Dilute Colloidal Suspensions. Molecular Physics, 61, 943-952. http://dx.doi.org/10.1080/00268978700101571

[24] Sood, A.K. (1987) Light Scattering from Colloids. Hyperfine Interactions, 37, 365-384. http://dx.doi.org/10.1007/BF02395721

[25] Ornstein, L.S. and Zernike, F. (1914) Accidental Deviations of Density and Opalescence at the Critical Point of a Single Substance. Proceeding of Akademic Science (Amsterdam), 17, 793-806.

[26] Hayter, J.B. and Hansen, J.P. (1982) The Structure Factor of Charged Colloidal Dispersions at Any Density. Institut Laue-Langevin Report, No. 82HA14T.

[27] Hayter, J.B. (1979) A Program for Fast Bi-Directional Transform between $G(R)$ and $S(Q)$. Institut Laue-Langevin Report, No. 79HA48S.

[28] Schaefer, D.W. (1977) Colloidal Suspensions as Soft Core Liquids. The Journal of Chemical Physics, 66, $3980-3984$. http://dx.doi.org/10.1063/1.434449

[29] Pieranski, P. (1983) Colloidal Crystals. Contemporary Physics, 24, 25-73. http://dx.doi.org/10.1080/00107518308227471

[30] Wagner, N.J., Krause, R., Rennie, A.R., Aguanno, B.D. and Goodwin, J. (1991) The Microstructure of Polydisperse, Charged Colloidal Suspensions by Light and Neutron Scattering. The Journal of Chemical Physics, 95, 494-508.

[31] Pandey, R.K. (2014) Comparison of Structure Factors Obtained with Sogami-Ise Potential and DLVO Potential for $\mathrm{H}^{+}-\mathrm{He}^{++}$Mixtures. SOP Transactions on Theoretical Physics, 1, 73-81.

[32] Pandey, R.K. (2014) Structure Factor of Suspended Polystyrene Macroions Using Sogami-Ise Potential. Journal of Physics \& Astronomy, 3, F67-F70. 